\title{
Malaria Parasite Infection and its Effect on Packed Cell Volume among Pregnant Women in Zaria, Nigeria
}

\author{
Obed Abigail, Maryam Aminu and Umar Abdullahi* \\ Department of Microbiology, Faculty of Life Sciences, Ahmadu Bello University, Zaria, Nigeria

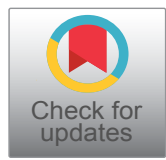

*Corresponding author: Umar Abdullahi, Department of Microbiology, Faculty of Life Sciences, Ahmadu Bello University, Zaria, Nigeria

\begin{abstract}
Malaria in pregnancy is a major public health challenge and priority, because it is a health risk for the mother and her fetus. This study was aimed at determining the prevalence of malaria parasites and its effect on packed cell volume (PCV) among pregnant women attending antenatal clinic in Zaria. A total of 102 pregnant women on ante-natal visit in three different hospitals were enrolled and questionnaires were administered to them to obtain data on some risks and sociodemographic factors that predispose to malaria. Blood samples $(2 \mathrm{ml}$ each) were collected from the women and examined microscopically for malaria parasites using thin and thick blood smears. Colored parasitology atlases were used as guide in identification. Packed Cell Volume (PCV) was determined using the microhaemoparasites centrifuge technique. Statistical analysis was done using IBM SPSS version 21.0 and $P$ value $\leq 0.05$ was considered significant. Malaria parasite was detected in $23.5 \%$ (24/102) of the women. Plasmodium falciparum was the only occurring Plasmodium specie detected. Pregnant women within the age group $36-40$ years had the highest infection with Plasmodium falciparum $(42.9 \%: 3 / 7)$, while those in age group $21-26$ years were the least infected $(12.5 \%: 3 / 24)$. Pregnant women in their third trimester were most infected with Plasmodium falciparum (27.6\%:8/29) than women in other trimesters. Women that had PCV lower than 30 and were considered anemic had higher percentage of the parasite $(42.9 \%: 12 / 28)$ than those with normal PCV (16.2\%:12/74). Nonuse of mosquito nets, presence of stagnant water in residential area and PCV were factors find to predispose to malaria in the study. It is recommended that pregnant women undergo regular assessment on malaria parasite infection so that early attention may be given to infected ones in order to have safe delivery.
\end{abstract}

\section{Keywords}

Malaria, Plasmodium falciparum, Pregnant women, PCV

\section{Introduction}

Malaria has always been the subject of research for medical practitioners from time immemorial. According to World Health Organization [1], pregnant women, children and immune-compromised individuals have the highest morbidity and mortality rate, and Africans bears the heaviest burden of malaria infection [1]. Malaria parasite belongs to the genus plasmodium. Plasmodium is the only genus belonging to the family plasmodidae, order Haemosporida, class coccidae, phylum sporozoa. The genus contains over 125 species that cause malaria in reptiles, birds and mammals [2]. Plasmodium falciparum and Plasmodium vivax are most widely spread than Plasmodium malariae and Plasmodium ovale [1]. The most common cause of malaria in the hotter and humid regions of Africa is Falciparum [1].

Human malaria parasite lives in the red blood cells and is transmitted by the female Anopheles mosquito. Malaria is a disease with major health problems that has attracted global concerns; hence it is regarded as the most important parasitic disease [3]. Malaria is most prevalent where there is poverty and where methods of disease identification, documentation and reporting are weak [4]. WHO [1] reported that in 2017, an estimated 219 million cases of malaria occurred worldwide $195 \%$ confidence interval $\mathrm{Cl}$ : 203-262 million), compared with 239 million cases in 2010 (95\% Cl: 219-285 million) and 217 million cases in 2016 (95\% Cl: 200-259 million). Although there was an estimated 20 million fewer malaria cases in 2017 than in 2010, data for the period 2015-2017 highlight that no significant progress

Citation: Abigail O, Aminu M, Abdullahi U (2021) Malaria Parasite Infection and its Effect on Packed Cell Volume among Pregnant Women in Zaria, Nigeria. Int J Trop Dis 4:055. doi.org/10.23937/2643461X/1710055

Accepted: December 08, 2021; Published: December 10, 2021

Copyright: (C) 2021 Abigail O, et al. This is an open-access article distributed under the terms of the Creative Commons Attribution License, which permits unrestricted use, distribution, and reproduction in any medium, provided the original author and source are credited. 
in reducing global malaria cases was made in this timeframe.

In Nigeria, there are over 100million people at risk of malaria every year and it is estimated that about $50 \%$ of the adult population experience at least one episode yearly [1]. According to reports by WHO [1], fifteen countries in sub-Saharan Africa and India carried almost $80 \%$ of the global malaria burden. Five countries accounted for nearly half of all malaria cases worldwide: Nigeria (25\%), Democratic Republic of the Congo (11\%), Mozambique (5\%), India (4\%) and Uganda (4\%).

Children aged under 5 years are the most vulnerable group affected by malaria. In 2017, they accounted for $61 \%(266,000)$ of all malaria deaths worldwide [1].

The WHO African Region accounted for $93 \%$ of all malaria deaths in 2017. Although the WHO African Region was home to the highest number of malaria deaths in 2017, it also accounted for $88 \%$ of the 172,000 fewer global malaria deaths reported in 2017 compared with 2010 [1]. Malaria causes a lot of debilitating effect in adults and the yearly economic loss due to malaria in Nigeria has been put at 132 million Naira comprising cost of treatment and transport to source of treatment, loss of man-powers, absenteeism from places of work and other indirect cost [5]. In 2017 [1], an estimated US\$ 3.1 billion was invested in malaria control and elimination efforts globally by governments of malaria endemic countries and international partners - an amount slighter higher than the figure reported for 2016. Nearly three quarters (US\$ 2.2 billion) of investments in 2017 were spent in the WHO African Region, followed by the WHO regions of South-East Asia (US\$300 million), the Americas (US\$200 million), and the Eastern Mediterranean and the Western Pacific (US\$100 million each).

A large proportion of severe malaria illness and deaths occur in people's home without coming to the attention of a formal health service [4]. WHO [1] reported that, between 2010 and 2017, estimated deaths due to malaria in African region declined from $555,000-403,000$. Malaria in pregnancy is a major public health challenge and a priority for the Roll back malaria partnership, because it is a health risk for the mother, her fetus and the neonate [5].

Most cases of malaria in pregnancy in areas of stable malaria transmission are asymptomatic [6]. Depending on the endemicity of malaria in an area, it can be expected that $1-50 \%$ of pregnant women may carry malaria parasitaemia especially in the placenta, without noticing it [6]. This is attributed to the anti-disease immunity acquired during previous exposure that protects against clinical malaria [7]. Pregnant women are three times more likely to suffer from severe disease as a result of malaria infection compared with their nonpregnant counterparts, and have a mortality rate that approaches $50 \%$ [8]. The symptoms and complications of malaria during pregnancy differ with the intensity of malaria transmission and the level of immunity the pregnant woman has acquired [9].

All pregnant women, regardless of the number of times they have been pregnant, are highly vulnerable to malaria [10]. Age has also been implicated as epidemiological studies have shown that malaria in pregnancy is more prevalent in younger than older age groups [11]. Currently the susceptibility of plasmodium parasitaemia has been linked to the level of antibodies to placental sequestrated parasites [12]. These parasites preferentially adhere to chondroitin dilatedreceptors (CSA) expressed by the syncytiophoblasts in the placenta [13].

In areas of Africa with stable malaria transmission, $P$. falciparum infection accounts for $99.7 \%$ of case incidence rate, $93 \%$ of all deaths $(403,000$ deaths in Africa) and $19 \%$ malaria mortality rate in Nigeria (Deaths per 100,000 populations at risk) [1]. In 2016, an estimated 92,699 post-neonatal deaths due to malaria was recorded in Nigeria [14].

Severe malaria in late pregnancy is a devastating and fulminant disease with a high mortality for both mother and fetus that is very difficult to manage, requiring close has on between physicians, Obstetricians and pediatrician. The case fatality is substantially higher in the second half of pregnancy (Because of the changes in women's immune systems during pregnancy and the presence of placenta with new places for parasites to bind, pregnant women lose some of their immunity to malaria infection) than in non-pregnant adults, and with quinine treatment often reached $50 \%$; while pregnant women diagnosed with uncomplicated malaria caused by chloroquine-resistant $P$. falciparum, women in the second and third trimesters can be treated with artemether-lumefantrine, and for all trimesters, mefloquine or a combination of quinine sulfate and clindamycin is recommended [1].

Antenatal clinic visit is a key for delivery of the prevention package for pregnant women. Studies have shown that $40 \%$ of pregnant African women present for the first time to antenatal clinics in the second trimester of their pregnancy [15]. Use of long lasting insecticide and nets also help in preventing malaria. In areas with moderate or high malaria transmission in Africa, intermittent preventive treatment in pregnancy (PTP) with sulphadoxine-pyrimethamine (SP) as part of antenatal care services, prompt diagnosis and effective treatment of malaria infections [10]. Considering the paucity of scientific investigations on the effects of malaria parasites on hematological profile of pregnant women.

This study was carried out to determine the prevalence of malaria parasites and its effect on 
packed cell volume among pregnant women attending antenatal clinics in Zaria.

\section{Materials and Methods}

\section{Study area and population}

The study was hospital based that employed the random sampling and conducted purposively and consecutively. The study was conducted in Zaria at antenatal clinics of Ahmadu Bello University Medical Centre, Al-Madina Clinic and Salamah Hospital. The study was carried out among pregnant women that were attending these antenatal clinics who consented to participate in this study regardless of the age and trimester of pregnancy. An introduction letter was collected from Department of Microbiology, Ahmadu Bello University Zaria and Ethical approval was obtained from the Ethical Committee of the selected hospitals.

\section{Collection of blood samples}

Prior to sample collection, structured questionnaires were administered to the pregnant women on antenatal clinic to obtain information on age, socio-demographic factors and risk factors among the pregnant women. The samples were collected between June and July 2018 from 102 pregnant women that satisfied the inclusion criteria. About $2 \mathrm{ml}$ of venous blood was collected by a laboratory technician using $5 \mathrm{ml}$ needle and syringe and was immediately transferred into a well labeled ethylene diamine tetra acetic acid (EDTA) bottles. The samples were transported to the laboratory of Microbiology Department, Ahmadu Bello University Zaria for further analysis. Only one blood sample per patient was collected.

\section{Laboratory analysis of blood samples}

All the blood samples were screened for malaria by microscopy using Thin and Thick film. The PCV of blood sample was determined by microhaematocrit centrifuge technique.

Thin and thick blood smear examination: For the thin smear a drop of blood was placed on a pre-cleaned labeled slide, near its frosted end. Another slide was placed at angle $30^{\circ}-45^{\circ}$ up to the drop; allowing the drop to spread along the contact line of the 2 slides. The upper slide was quickly pushed towards the unfrosted end of the lower slide. The thin smears were allowed to dry and fixed by dipping it in absolute methanol and stained with $10 \%$ Giemsa stain. For the thick smear small drop of blood of blood was used and was stained the same way with the thin blood film. It was washed and allowed to dry. The dry slides were examined under the microscope (100x oil immersion).

Packed cell volume determination: The capillary tube was filled with blood to about $2 / 3$ to $1 / 4$ of the capillary tube. The other end of the capillary tube was sealed with a plastic sealant. The filled tubes were

Table 1: Prevalence of $P$. falciparum infection from pregnant women attending ante-natal clinic from various Hospitals in Zaria.

\begin{tabular}{|l|l|l|l|}
\hline Hospital & Total & Positive & \% Prevalence \\
\hline Al-Madina Clinic & 20 & 8 & 40 \\
\hline ABU Medical Centre & 79 & 16 & 20.3 \\
\hline Salamah Hospital & 3 & 0 & 0 \\
\hline Total & 102 & 24 & 23.5 \\
\hline
\end{tabular}

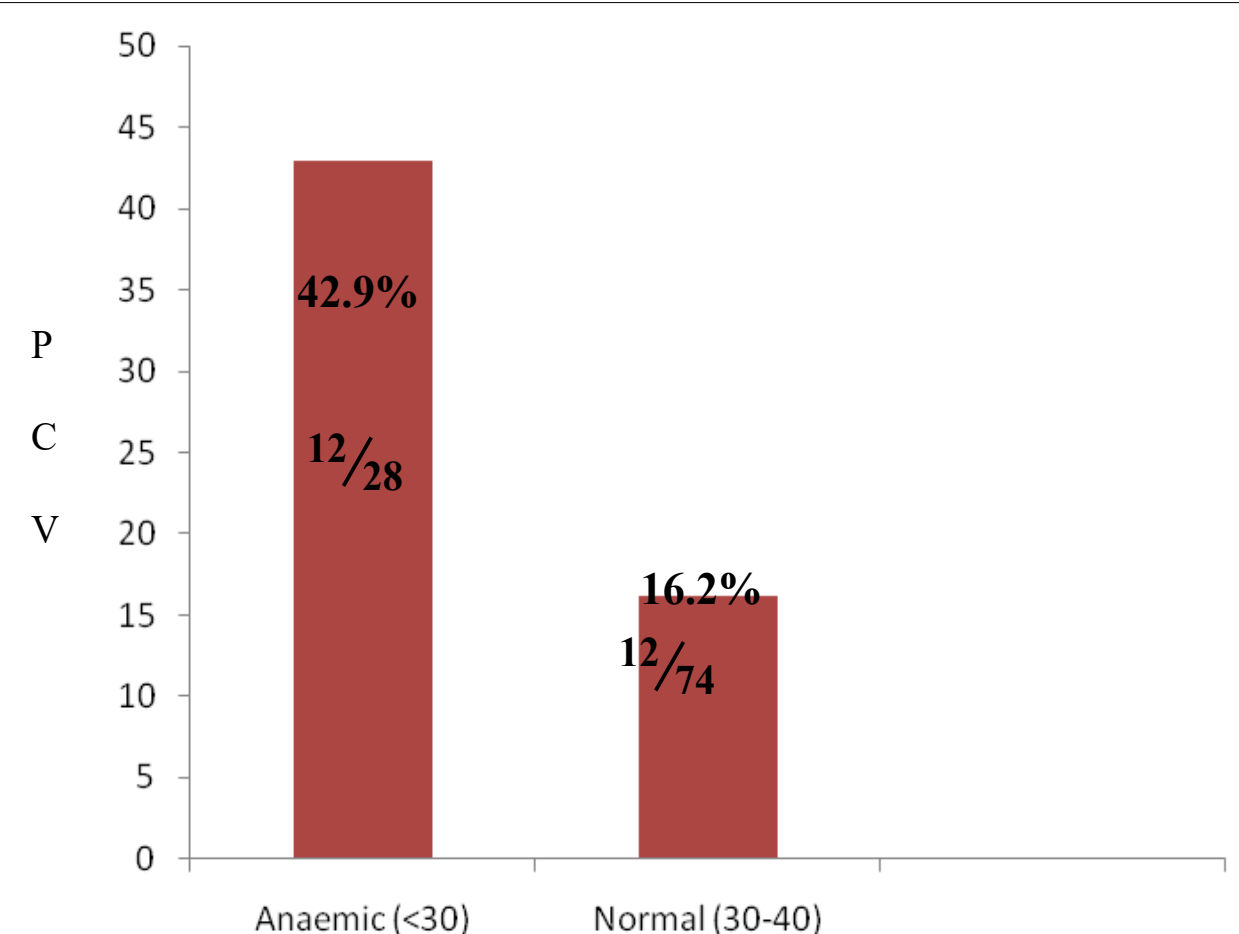

Figure 1: Prevalence of malaria parasite by PCV Level. PCV: Packed Cell Volume 
placed in the radial grooves of the centrifuge with the sealed end towards the outer rim gasket. The centrifuge was set at 10,000 to 12,000 revolutions per minute (rpm) for 5 minutes to completely pack the red cells. The tube was placed in the microhematocrit reader and the hematocrit will be read following the manufacturer's instructions [16].

\section{Data analysis}

Data from questionnaire and laboratory results were entered into MS Excel and was subjected to ANOVA, chi square, Spearman Correlation and Odds Ratio analyses using IBM SPSS version 21.0. $\mathrm{P} \leq 0.05$ at $95 \%$ confident interval $(\mathrm{Cl})$ was considered significant.

\section{Result}

The result of the study shows that out of the 102 blood samples collected and examined microscopically, 24 were found infected with Plasmodium falciparum giving a prevalence of $23.5 \%$ (23/102). Table 1 shows the prevalence of $P$. falciparum infection from pregnant women attending some ante-natal clinic from various hospitals in Zaria. A total of 20 samples were collected from Al-Madina Clinic, 79 samples from ABU Medical Centre and 3 samples from Salamah Hospital. The highest Plasmodium prevalence was seen from samples collected from Al-Madina Clinic (8/20:40\%). While no parasite was detected from samples collected from Salamah Hospital.

A total of $12(42.9 \%)$ of these women were anemic $(\mathrm{PCV}<30)$ and $12(16.2 \%)$ were normal (PCV between 31-40) (Figure 1).

Table 2 shows the distribution of malaria parasites according to age group of pregnant women attending some ante-natal clinic in Zaria. The highest prevalence was seen among women in the age group 36-40 years $(3 / 7: 42.9 \%)$ and the lowest was seen among women in the age group $21-25$ years (3/24:12.5\%). The highest mean number $(+10)$ of infected RBC per volume $(\mu l)$ was seen among pregnant women in the age group 26-30 years. There was no statistically significant association between the presence of malaria parasite and the age group of the pregnant women $\left(\chi^{2}=4.281, \mathrm{df}=4, \mathrm{p}=\right.$ $0.369)$.

Table 3 shows the mean PCV of pregnant women by gestational age (Trimester) attending some antenatal clinic in Zaria. The highest prevalence was seen in pregnant women in their second Trimester $27.6 \%$ $(8 / 24)$ compared to women in their third trimester who had a lowest prevalence of $29.9 \%$ (14/64). There was no statistically significant association between the mean PCV of pregnant women by trimester $\left(\chi^{2}=0.3771, \mathrm{df}=\right.$ $2, p=0.831)$.

Table 4 shows some sociodemographic factors associated with malaria among pregnant women attending some ante-natal clinic in Zaria. The highest prevalence of $28.9 \%(11 / 38)$ was seen in women who were multigravidae while the lowest was seen in women who were primigravidae $7 / 36(19.4 \%)$ but the difference was not statistically significant $\left(\chi^{2}=1.022\right.$, df $=2, p=0.600$ ). The prevalence of malaria parasites in level education occur most in the women that stopped at secondary school $11 / 33(33 \%)$ but was not statistically significant $\left(\chi^{2}=2.695, d f=2, p=0.260\right)$. Pregnant women that were unemployed had higher prevalence of $24.6 \%$ $(17 / 69)$ compare to those that were employed $21.2 \%$ (7/33) but the difference was not statistically significant $\left(\chi^{2}=0.146, d f=1, p=0.703\right)$.

Table 2: Distribution of malaria parasites according to age of pregnant women attending ante-natal clinic Zaria, Nigeria.

\begin{tabular}{|l|l|l|l|l|l|l|l|}
\hline $\begin{array}{l}\text { Age group } \\
\text { (years) }\end{array}$ & $\begin{array}{l}\text { Number } \\
\text { examined }\end{array}$ & $\begin{array}{l}\text { P. falciparum } \\
\text { positive (\%) }\end{array}$ & $\begin{array}{l}\text { Mean PCV } \\
\text { positive }\end{array}$ & $\begin{array}{l}\text { Mean PCV } \\
\text { Negative }\end{array}$ & \multicolumn{2}{l|}{$\begin{array}{l}\text { Intensity } \\
\text { Mean number of infected RBC per } \\
\text { volume }(\boldsymbol{\mu l )})\end{array}$} \\
\hline $16-20$ & 8 & & & + & ++ \\
\hline $21-25$ & 24 & $3(37.5)$ & 31.667 & 32.600 & 2 & 0 & 1 \\
\hline $26-30$ & 38 & $3(12.5)$ & 29.333 & 33.143 & 0 & 3 & 0 \\
\hline $31-35$ & 25 & $10(26.3)$ & 20.600 & 33.250 & 2 & 3 & 5 \\
\hline $36-40$ & 7 & $5(20.0)$ & 31.800 & 32.850 & 2 & 3 & 0 \\
\hline Total & 102 & $3(42.9)$ & 32.000 & 33.500 & 1 & 1 & 1 \\
\hline
\end{tabular}

Table 3: Mean PCV of pregnant women by Trimester attending ante-natal clinic in Zaria.

\begin{tabular}{|l|l|l|l|l|l|}
\hline Trimester & $\begin{array}{l}\text { Number } \\
\text { examined }\end{array}$ & Mean PCV (\%) & $\begin{array}{l}\text { P. falciparum } \\
\text { Percentage }\end{array}$ & $\begin{array}{l}\text { positive } \\
\text { prevalence }\end{array}$ & 22.2 \\
\hline First & 9 & 31.778 & 2 & 27.6 \\
\hline Second & 29 & 32.103 & 8 & 21.9 \\
\hline Third & 64 & 32.781 & 14 & 23.5 \\
\hline Total & 102 & 32.500 & 24 & \\
\hline
\end{tabular}


Table 4: Some socio-demographic factors associated with malaria among pregnant women attending ante-natal clinic in Zaria.

\begin{tabular}{|c|c|c|c|c|}
\hline Variable & Number examined & $\begin{array}{l}\text { P. falciparum } \\
\text { prevalence }\end{array}$ & $\begin{array}{l}\text { Percentage } \\
\text { positive (\%) }\end{array}$ & P value \\
\hline \multicolumn{5}{|l|}{ Gravidity } \\
\hline Multigravidae & 38 & 11 & 28.9 & 0.600 \\
\hline Second gravidae & 28 & 6 & 21.4 & \\
\hline Primigravidae & 36 & 7 & 19.4 & \\
\hline \multicolumn{5}{|l|}{ Marriage type } \\
\hline Monogamy & 82 & 17 & 20.7 & 0.177 \\
\hline Polygamy & 20 & 7 & 35.0 & \\
\hline \multicolumn{5}{|c|}{ Educational status } \\
\hline Primary & 4 & 1 & 25.0 & 0.260 \\
\hline Secondary & 33 & 11 & 33.3 & \\
\hline Tertiary & 65 & 12 & 18.5 & \\
\hline \multicolumn{5}{|l|}{ Occupation } \\
\hline Employed & 33 & 7 & 21.2 & 0.703 \\
\hline Unemployed & 69 & 17 & 24.6 & \\
\hline Total & 102 & 24 & 23.5 & \\
\hline
\end{tabular}

Table 5: Effect of malaria parasites on packed cell volume among pregnant women attending some ante-natal clinic in Zaria.

\begin{tabular}{|c|c|c|c|c|c|}
\hline \multirow[t]{2}{*}{ PCV Status } & \multirow[t]{2}{*}{ Number examined } & \multirow[t]{2}{*}{$\begin{array}{l}\text { P. falciparum } \\
\text { positive (\%) }\end{array}$} & \multicolumn{3}{|c|}{$\begin{array}{l}\text { Intensity mean number of infected RBC per } \\
\text { volume }(\mu \mathrm{l})\end{array}$} \\
\hline & & & + & ++ & +++ \\
\hline Anaemic $(<30)$ & 28 & $12(42.9)$ & 3 & 6 & 3 \\
\hline Normal (31-40) & 74 & $12(16.2)$ & 4 & 4 & 4 \\
\hline Total & 102 & $24(23.5)$ & 7 & 10 & 7 \\
\hline
\end{tabular}

Table 6: Risk factors for malaria infection among pregnant women attending some ante-natal clinic in Zaria.

\begin{tabular}{|c|c|c|c|c|c|c|}
\hline \multirow[t]{2}{*}{ Variable } & \multirow[t]{2}{*}{ Number examined } & \multirow[t]{2}{*}{$\begin{array}{l}\text { P. falciparum } \\
\text { positive (\%) }\end{array}$} & \multirow[t]{2}{*}{ OR $(95 \% \mathrm{Cl})$} & \multicolumn{3}{|c|}{$\begin{array}{l}\text { Intensity mean number of } \\
\text { infected RBC per volume ( } \mu \mathrm{l})\end{array}$} \\
\hline & & & & + & ++ & +++ \\
\hline \multicolumn{7}{|c|}{ Presence of stagnant water } \\
\hline No & 59 & $8(13.6)$ & 3.778 (1.43-9.95) & 3 & 2 & 3 \\
\hline Yes & 43 & $16(37.2)$ & & 4 & 8 & 4 \\
\hline \multicolumn{7}{|c|}{ Use of mosquito net } \\
\hline Yes & 74 & 22(29.7) & $5.500(1.20-25.20)$ & 6 & 9 & 7 \\
\hline No & 28 & $2(7.1)$ & & 1 & 1 & 0 \\
\hline Total & 102 & $24(23.5)$ & & 7 & 10 & 7 \\
\hline
\end{tabular}

Table 5 shows the effect of malaria parasites on packed cell volume among pregnant women attending ante-natal clinics in Zaria. The highest prevalence was seen in pregnant women that were anaemic $(<30)$ $12 / 28$ (42.9\%) while those with normal PCV had the least $12 / 74$ (16.2\%). There was statistically significant association between the presence of malaria parasite and the level of PCV $\left(\chi^{2}=8.013, d f=1, p=0.005\right)$.

Table 6 shows some risk factors for malaria infection among pregnant women attending ante-natal clinics in Zaria. Higher prevalence was recorded among pregnant women who live in houses with stagnant water in the environment (37.2\%:16/43) and lower prevalence was seen among pregnant women who live in houses without stagnant water in the environment (13.6\%:8/59). There was a significant association between the presence of stagnant water in the environment that the pregnant women live and the presence of the parasite $\left(\chi^{2}=7.732\right.$, $\mathrm{df}=1, \mathrm{p}=0.005$ ). Women who lived in houses with stagnant water was at least 4 times tendency to be infected with the parasite $(\mathrm{OR}=3.778 ; 95 \% \mathrm{Cl} 1.434$ 9.950). Higher prevalence was seen among women that use mosquito net (29.7\%:22/74) than women that did not (7.1\%:2/28). There was significant association between the use of mosquito net by the pregnant women and the presence of the parasite $\left(\chi^{2}=5.760, \mathrm{df}\right.$ 
Table 7: Correlation of intensity of malaria parasites with packed cell volume among pregnant women attending ante-natal clinics in Zaria.

\begin{tabular}{|l|l|l|l|l|l|}
\hline Parameter & Mean & SD & PCV & Intensity & p-value \\
\hline PCV & 32.500 & 3.0499 & 1 & -0.314 & 0.001 \\
\hline Intensity & 0.471 & 0.9303 & -0.134 & 1 & 0.001 \\
\hline
\end{tabular}

Spearman Correlation (R): -0.314 Weak negative Correlation; PCV: Packed Cell Volume; SD: Standard Deviation

$=1, p=0.016)$. Women who use mosquito net was at least 6 times tendency to be infected with the parasite ( $O R=3.778 ; 95 \% \mathrm{Cl} 1.200-25.202$ ).

Table 7 shows the correlation of intensity of malaria parasites with packed cell volume among pregnant women attending ante-natal clinic in Zaria. Packed Cell Volume has a very weak negative correlation $(-0.314)$ with increasing intensity of $P$. falciparum parameter in blood samples pregnant women.

\section{Discussion}

The result obtained in this study show $23.5 \%$ of the populations of pregnant women examined were positive for malaria parasite. This shows that pregnant women in the study area are exposing to malaria parasite and this infection could lead to still birth, spontaneous abortion, maternal anemia, placental parasitaemia and death of the mother. The relatively lower prevalence rates of malaria infection among these pregnant women may not be as a result of the development of higher levels of the acquired anti-malaria immunity among them. But a more plausible explanation for this lower prevalence rate could be attributed to increased malaria awareness among the women as has been seen in many endemic areas of the sub-Sahara Africa. It could also be due to intensified efforts of various health authorities at the local, regional and national levels in the control and prevention of malaria in pregnancy.

The world Health Organization currently recommend that women in areas of high malaria transmission in Africa receive intermittent treatment with an effective anti-malaria drug at regularly scheduled Anti-Natal Care (ANC) visits after quickening is being implemented in many malaria endemic areas [17]. The result of this study differs from that carried out in Kano [18] and Abakaliki [17] with higher prevalence of $80.9 \%$ and $42 \%$ respectively.

In relation to parity, the prevalence of malaria parasite was insignificantly higher among the multigravidae $(28.9 \%)$ than the second gravidae and primigravidae $(p>0.5)$. Malaria infection is highly controlled by the immune system and as such may be clinically unrecognized unless diagnosed or investigated making pregnant women to be particularly at risk [19]. The results were not in concordance with the findings from similar studies conducted in Kano [18] in the view that parasitaemia was significantly higher in primigravidae than in multigravidae. The result however contrasts that of Dicko, et al. [20] that shows that the multigravidae were the most susceptible group and that the protective immunity in pregnancy is not a function of parity.

Pregnant women within age group of 36-40 years had the highest prevalence of $P$. falciparum. The reason for the high prevalence of infection among the group may be because of the relative small number of pregnant women examined in the group or may be due to the direct effect of parity, because a high percentage of the group may be older but younger first time mothers. This study agrees with the result of the study carried out in Ado-Ekiti [21] who also obtained the highest prevalence of plasmodium infection among women in age group 36-40 years.

Pregnant women with PCV $<30$ had higher prevalence of $P$. falciparum and the difference in prevalence was statistically significant. The drop in PCV among the infected pregnant women could be as a result of extravascular destruction of both parasitized and unparasitized erythrocytes that takes place in the spleen during malaria infection [12]. It has been shown that malaria often causes anemia with decreased uterine activity and low birth weight among others [22]. Other studies fairly similar to the present study found higher prevalence of $92.1 \%$ and $47 \%$ for malaria parasite among pregnant women in Akure, Ondo state [23] and Benue State [23].

Plasmodium falciparum was the only specie of malaria parasite detected in the blood sample of the pregnant women. This finding is not surprising because this specie is considered to be the most predominant among the 4 plasmodium parasites [24,25]. All pregnant women who claimed to use mosquito nets in this study had higher prevalence of malaria parasite than those that did not. This may be due to improper use of mosquito net or response in the questionnaire was not true. Also the pregnant women may be exposing to malaria parasite before bedtime.

Unemployed pregnant women more infected with $P$. falciparum. This is probably due to staying late hours of the night carrying out businesses and consequently gets bitten repeatedly. However high prevalence among the unemployed has also been reported by Bishop and Aliyu [18].

Analysis of the results in relation to trimester showed that malaria parasite was detected with the highest prevalence among pregnant women with no statistically significant difference. It is established 
that immunosuppression is evident during the second trimester of pregnancy and this possibly results from the presence of high adrenal steroid levels as well as chorionic gonadotropin and fetoprotein and the blood, there may also be depression of the lymphocyte activity [17]. This finding agrees with that reported in the study carried out at Abakaliki, Southeast Nigeria Oliver, et al. [17], with highest occurance of the infection in second trimester but contrast the of the finding carried out in Kano [18], where the level of Plasmodium infection was higher in the third trimester.

\section{Conclusion}

Malaria parasite was detected in 24 (23.5\%) of 102 pregnant women and Plasmodium falciparum was the only Plasmodium specie found in the study. Women in the age group of 36-40 years had the highest prevalence of Malaria parasite. Women that were anemic $(<30)$ had higher prevalence than those with normal PCV (31-40) with the same intensity. Use of mosquito nets and presence of stagnant water were factors find to predispose to malaria in the study.

\section{Acknowledgements}

We appreciate the pregnant women that participated in this study. We also appreciate the hospital management for granting us the permission to conduct this study and the hospital staff for their supports.

\section{References}

1. World Health Organization (2018) World malaria report 2018.

2. Hommel M, Gilles HM (2006) Topley and Wilson's Microbiology and Microbial Infections: Malaria. (4th edn), Edward Amold Publishers Ltd.

3. Onolade OO (2003) Public health implications on pregnant women. American Journal Reproductive Health 7: 77-83.

4. World Health Organization (2014) Severe Malaria. Tropical Medicine \& International Health 19: 1-131.

5. Igbeneghu C, Odaibo AB (2013) Impact of acute malaria on some haematological parameters in a semi-urban community in Southwestern Nigeria. Acta Parasitologica Globalis 4: 01-05.

6. Center for Disease Control (2019) Malaria: Guidelines for clinicians; alternatives for pregnant women for treatment of severe malaria.

7. Staalsoe T, Shulman CE, Bulmer JN, Kawuondo K, Marsh $K$ et al. (2004) Variant surface antigen-specific IgG and protection against clinical consequences of pregnancyassociated plasmodium falciparum malaria. Lancet 363 : 283-289.

8. World Health Organization (2006) Guidelines for treatment of malaria. Geneva.

9. World Health Organization (2004) A strategic framework for malaria prevention and control during pregnancy in the African Region. Geneva.

10. World Health Organization (2017) Malaria in pregnant women.
11. Bouyou-Akotet MK, Lonete-Collard DE, Mabika-Manfoumbi M, Kendjo E, Matsiegui PB, et al. (2003) Prevalence of plasmodium falciparum infection in pregnant women in gabon. Malar J 2:18.

12. Elliot SR, Brennan AK, Beeson JG, Tadesse E, Molyneux $M E$, et al. (2005) Placental malaria induces variant-specific antibodies of the cytophilic subtypes immunoglobulin $\mathrm{G} 1$ (IgG1) and IgG3 that correlate with adhesion inhibitory activity. Infect Immun 73: 5903-5907.

13. Fried M, Nosten F, Brockman A, Brabin BJ, Duffy PE (1998) Maternal antibodies block malaria. Nature 395: 851-852.

14. UNICEF (2018) Estimates of child cause of death, malaria 2018.

15. Norgan AP, Arguello HE, Sloan LM, Fernholz EC, Pritt BS (2013) A method for reducing the sloughing of thick blood films for malaria diagnosis. Malaria Journal 12: 231.

16. Cheesbrough M (2010) District laboratory practice in tropical countries. Part I, (2 ${ }^{\text {nd }}$ edn), Cambridge, UK, 195216.

17. Odikamnoro O, Iganga A, Ozowara NL, Okoh N (2014) Prevalence of malaria among pregnant mothers and possible relationship to parity in Abakaliki, Southeast Nigeria. European Journal of Experimental Biology 4: 1519.

18. Bishop HG, Abdulfatah GA (2017) Menace of Haemoparasitic infections in pregnant women attending Unguwa Uku clinic and maternity hospital, Kano-Nigeria. International Journal of Microbiology and Biotechnology 2: 65-74.

19. Odaibo SF (2005) Health watch. 53: 5-15.

20. Dicko A, Mantel C, Aly TM, Doumbia S, Diallo M, et al. (2003) Risk factors for malaria infection and anaemia for pregnant women in the Sahel Area of Bandiagara, Mali. Acta Trop 89: 17-23.

21. Nyamngee LD, Edungbola AH, Akanbi AA (2014) Malaria parasitaemia among pregnant women in Ado Ekiti Nigeria Global Journal of Biology, Agriculture and prevalence of malaria among pregnant mothers and possible relationship to parity in Abakaliki, Southeast Nigeria. 4: 15-19.

22. Steketee RW, Wirima JJ, Campbell CC (1996) Developing effective strategies for malaria prevention programs for pregnant African women. Am J Trop Med Hyg 55: 95-100.

23. Abe AF, Olusi TA (2014) Seroprevalalence of malaria parasite infection among pregnant women attending two tertiary health facilities in Akure Ondo state Nigeria. J Bacteriol Parasitol 5: 1-6.

24. Centers for Disease Control and Prevention (2012) Malaria treatment (United States).

25. World Health Organization (2003) Antenatal care in developing countries. Promises, achievements and missed opportunities: An analysis of levels, trend and differentials, 1990-2001. 